\title{
Hospital do Câncer de Barretos
}

\author{
Carlos Afonso Clara, MD, $\mathrm{PhD}^{1}$ \\ ${ }^{1}$ Departamento de Neurocirurgia, Hospital de Câncer de Barretos, \\ Barretos, SP, Brasil \\ Arq Bras Neurocir 2015;34:342.
}

Prezado Editor-Chefe Dr. Eberval Gadelha Figueiredo,

Referente ao artigo publicado na revista Brazilian Neurosurgery (volume 34, número 2, 2015): Remote Cerebellar Hemorrhage after Surgery for Spinal Column Tumor: An Unusual Cause of Impaired Consciousness.

Como é de seu conhecimento através do contato que fizemos com a Sociedade Brasileira de Neurocirurgia (SBN), o autor publicou um caso tratado no Hospital de Câncer de Barretos (HCB) sem o conhecimento e autorização da Instituição e do Departamento de Neurocirurgia. O projeto não foi avaliado ou aprovado pelo Comitê de Ética do HCB. Não foi obtido termo de consentimento do paciente. $\mathrm{O}$
Address for correspondence Carlos Afonso Clara, MD, PhD,

Departamento de Neurocirurgia, Hospital de Câncer de Barretos, Avenida 27, $n^{\circ}$ 956, Barretos, SP, Brasil CEP: 14784-400

(e-mail: carlosclara.neuro@gmail.com).

autor não é médico do $\mathrm{HCB}$, foi estagiário como médico observador por 90 dias em 2014. Os coautores também não são médicos do HCB e consta que desconheciam o envio da publicação. Acreditamos que houve um problema de ordem ética. Já sabemos das providências que estão sendo tomadas pela SBN e pela revista para solucionar esse caso.

Gratos pela atenção.

Carlos Afonso Clara, MD, PhD Departamento de Neurocirurgia, Hospital de Câncer de Barretos,

Barretos, SP, Brasil received

July 24,2015

accepted

August 18, 2015

published online

October 28, 2015
DOI http://dx.doi.org/

$10.1055 / \mathrm{s}-0035-1564420$.

ISSN 0103-5355.
Copyright $\odot 2015$ by Thieme Publicações License terms Ltda, Rio de Janeiro, Brazil 